\title{
Formation of Neutrophil Extracellular Traps against Infection and During Inflammation: Boon or a Bane?
}

\section{Sultan Z Alasmari*}

King Khalid University, Abha, Asir Region 61421, Saudi Arabia

*Corresponding author: Sultan Z Alasmari, Faculty of Applied Medical Sciences, King Khalid University, Abha, Asir Region 61421, Saudi Arabia, Tel: +966555757019; E-mail: szaher@kku.edu.sa

Received date: July 10, 2018; Accepted date: August 14, 2018; Published date: August 21, 2018

Copyright: ( 2018 Alasmari SZ. This is an open-access article distributed under the terms of the Creative Commons Attribution License, which permits unrestricted use, distribution and reproduction in any medium, provided the original author and source are credited.

\begin{abstract}
Neutrophils are the first line of innate immune defence against pathogens. They have three different processes for directly attacking microorganisms, including phagocytosis, degranulation, and forming neutrophil extracellular traps (NETs). The methods of degranulation and phagocytosis are well established. However, several recent studies have been conducted to understand the potential role of NETs in diseases. The involvement of NETs was shown in several conditions including infections and inflammation. This review will discuss the morphology and mechanisms of NET formation, as well as NET formation against infection and during inflammation.
\end{abstract}

Keywords: Neutrophils; Neutrophil extracellular traps (NETs); NET processes; Microorganisms; Autoimmune disorders

\section{Introduction}

In mammals, neutrophils are the most abundant type of white blood cell and are the first line of defence against pathogens. For decades, it has been known that neutrophils kill pathogens through two unique processes: phagocytosis and degranulation. However, Brinkmann et al. described a new third process [1]. They found that activated neutrophils undergo cell death distinct to programmed cell death dependent of NADPH oxidase and elastase to form a web-like structure. These web-like structures are called neutrophil extracellular traps (NETs) and are composed of histones H1, H2A, H2B, H3 and H4, neutrophil elastase, and myeloperoxidase [1]. Neutrophils form NETs to trap and kill microorganisms extracellularly and die following NET release. Yipp et al. revealed another novel mechanism of NET formation where activated neutrophils remain active and capable of chasing and engulfing pathogens following NET release [2]. This process involves integrins and toll-like receptors (TLRs) and is independent of NADPH and elastase. A third new process was demonstrated recently by Mohanty, which showed that saliva could trigger NET formation independent of NADPH, elastase, and integrins [3].

\section{NET Morphology}

Using high-resolution scanning electron microscopy (SEM), Brinkmann et al. found that NETs are composed of smooth strands and a globular domain [1]. The measurement of the threads shows diameters of 15 to $17 \mathrm{~nm}$ while the globular domains of around $25 \mathrm{~nm}$. Together, threads and globular domain could stretch and aggregate to create a cloud-like structure of NETs occupy space more significant than the cell they originated from by 10-15 fold [4]. However, Transmission electron microscopy (TEM) showed that NETs are not surrounded by membranes [1]. Further, an immunofluorescence study of NETs showed that NETs consist of azurophilic (primary) granules such as cathepsin G, neutrophil elastase, and myeloperoxidase.
Secondary and tertiary granules such as lactoferrin and gelatinase, respectively, were also found in NETs [1].

\section{Processes of NET Formation}

Neutrophils undergo various processes to perform NET formation or NETosis. First coined by Steinberg et al. the term NETosis describes neutrophil cell death leading to NET formation, which is the first process of NET release [5]. This form of NETosis was later termed suicidal NETosis [2]. In suicidal NETosis, NET formation takes over an hour for completion. On activation, the cells flatten and firmly attach to the substratum. Subsequently, the internal cell components progressively undergo changes. Lastly, the cells contract until the membrane ruptures leading to NET formation [4]. Suicidal NETosis is regulated by NADPH, myeloperoxidase, and elastase and is induced by microorganisms and chemical agents such as PMA [1,6,7]. Yipp et al. demonstrated a new NET formation process where anucleated neutrophils continued their normal cellular functions, such as leukocyte recruitment, chemotaxis, and phagocytosis following NET release [2]. This process, termed vital NETosis, is stimulated by bacteria and forms NETs rapidly $[2,8]$. There are three fundamental differences between vital NETosis and suicidal NETosis. These differences include: 1 . The nature of stimuli and timing of NET release. For example, chemical stimuli induce suicidal NETosis and requires hours for neutrophils to form NETs while bacteria induces vital NETosis and is rapid $[1,8] ; 2$. The functional capacity of neutrophils during NET release. For instance, Kubes' lab developed a method to visualise NET release in a mouse model of a bacterial skin infection using intravital confocal microscopy and found that neutrophils release NETs and remain functional following NET release [2]; 3. The processes employed to make and release NETs. For instance, in suicidal NETosis, neutrophil activation by PMA results in raf-MEK-ERK stimulation and, subsequently, activation of NADPH oxidase. Decondensation of chromatin is mediated by neutrophil elastase (NE) and myeloperoxidase (MPO) leading to a mixture of intracellular components that extrude to the extracellular space. In contrast, vital NETosis requires DNA movement from within the nucleus to the extracellular space without cell membrane rupture [2]. Further, 
Mohanty et al. discussed a new third novel process of NET release providing antimicrobial defence at oral mucosa elicited by sialyl LewisX-1-selectin signaling [3]. Neutrophils exposed to bacteria release NETs by a process involving integrins and toll-like receptors (TLRs) [2,8-10]. Despite microorganisms being potent inducers of NETs by processes dependent on $\beta 2$-integrins, saliva induces NETs in the absence of $\beta 2$-integrin. Sialyl LewisX, which is a ligand for l-selectin is presented in the mucin of the saliva and is shown to play a role in NET formation [3]. Neutrophil incubation with saliva treated with sialidase an inhibitor of Sialyl LewisX fails to release NETs, which confirms the role of sialyl LewisX in NET formation, and oral NETs are elicited by low sialyl LewisX concentration [3]. Interestingly, vital NETosis and saliva-induced NETosis have been shown to undergo NET formation rapidly. The only difference between vital NETosis and saliva-induced NETosis occurs in the intercellular nuclear events. In vital NETosis, nuclear disruption occurs in the late stage of NET release and results in free nucleus neutrophils capable of chasing and engulfing microorganisms. In contrast, saliva-induced NETosis shows nuclear disruption in the early stage of NET release.

\section{NETs and Microorganisms}

NETs trap microorganisms extracellularly and disarm them using enzymes (MPO,proteases, lysosome), histones, antimicrobial peptides (BPI, defensins), and ion chelators (calgranulin) $[1,4,11]$. This process prevents the spread of microbes and protects the body from serious complications. Multiple gram-positive and negative bacteria, protozoa, and viruses stimulate neutrophils to form NETs.

\section{NETs and Bacteria}

Several gram positive and negative bacteria have been shown to form NETs. Neutrophils exposed to Staphylococcus aureus form rapid NETs without lysing [8]. Rapid NETs contribute to trapping and killing $S$. aureus NET formation. $S$. aureus is independent of reactive oxygen species (ROS) produced by NADPH oxidase. Streptococcus pneumoniae is another gram-positive bacterium that induces NET formation [12]. Unlike $S$. aureus, $S$. pneumoniae is trapped by NETs but not killed. The endA gene encodes the membrane nuclease of Streptococcus pneumoniae and is necessary for DNA uptake in genetic transformation and degrades the extracellular DNA of NETs that help trap $S$. pneumoniae to escape NETs $[12,13]$. Mice injected intranasally with a sublethal dose of Klebsiella pneumoniae formed a web-like structure composed of histone and myeloperoxidase consistent with NET formation in the lungs [7]. Listeria monocytogenes are another gram-positive bacteria shown to induce NET formation $[14,15]$. Further known to induce NET formation is Mycobacterium tuberculosis, which is one of the most common causes of death worldwide [16]. Interestingly, M. tuberculosis genotypes can be trapped but not killed by NETs [16]. Further, NET formation is triggered by Aspergillus fumigatus [17]. However, NETs do kill $A$. fumigatus hyphae [17]. Candida albicans and Candida glabrata have also been known to induce the release of NET formation $[18,19]$.

Shigella flexneri, a gram-negative bacterium is an inducer of NET formation [1]. Haemophilus influenzae, another gram-negative bacteria, is divided into typeable and nontypeable strains on the presence or absence of a polysaccharide capsule [20]. The nonencapsulated forms nontypeable Haemophilus influenzae (NTHI), which is a serious pathogen that causes several infections such as Haemophilus respiratory infections, sinusitis, and otitis media [20,21]. Neutrophils exposed to NTHI form NETs mediated by NHTI lipooligosaccharides (LOS) [22]. Interestingly, NET formation is triggered and degraded by Yersinia enterocolitica [23]. Neutrophils incubated with the serotype O:3, O:8, and O:9 of Yersinia enterocolitica induce NET formation. The O:3 strain is the most potent inducer [23]. In contrast, the strongest degradation was observed in $\mathrm{O}$ : 8 and O:9 strains. Periodontitis is a serious disease characterised by the destruction of tooth-supporting tissues. The gram-negative anaerobe, Porphyromonas gingivalis, is the keystone species for developing chronic periodontitis [24]. P. gingivalis has also been found as an inducer of NET formation [25]. Vibrio cholera is another gramnegative bacterium; it is the main cause of watery diarrhoea. $V$. cholera induces NET formation and later degrades it using extracellular nucleases Dns and Xds [26].

\section{NETs and Viruses}

Several researchers have investigated the role of NET formation during virus infection. In one study, purified neutrophils incubated with the influenza A virus formed a cloud-like structure stained with SYTOX green dye. SYTOX stained extracellular DNA but not the DNA of intact cells [27]. Interestingly, NET formation was not shown when neutrophils were exposed to the dengue virus serotype-2 (DENV-2). However, DENV-2 inhibits $80 \%$ of NET-produced PMA stimulus [28]. The interaction of NET formation with HIV-1 has also been investigated. NETS are shown to trap HIV-1 and inhibit its dissemination using myeloperoxidase and $\alpha$-defensin [29]. Toll-like receptors TLR7 and TLR8 expressed on neutrophils recognise HIV-1 and induce reactive oxygen that triggers NET formation [29]. Respiratory syncytial virus (RSV) is a major cause of paediatric infections and leads to severe lower respiratory tract disease (LRTD) [30]. Neutrophils exposed to RSV form NETs to prevent the virus from spreading [30]. However, exaggerated NET formation during severe RSV-LRTD may lead to obstruction of the airway.

\section{NETs and Fungi}

NET formation has also been observed during fungal infections. Urban et al. found that Candida albicans, a common human pathogen, induces NET formation in vitro. NETs can kill the yeast form and hyphae cells of Candida albicans [18,31]. As mentioned above, NETs are composed of histones and granular proteins. The yeast form and hyphae cells were killed by the granular proteins of NETs but not histones [18]. Also, neutrophils release NETs when exposed to different strains of Aspergillus fumigatus morphotypes [32]. However, the amount of NET formation is morphotype and strain-dependent [32]. A recent in vivo study showed that NADPH oxidase is required for NET formation and the clearance of Aspergillus hyphae [33]. Cryptococcus is another pathogenic fungus. It is an opportunistic fungus in immunocompromised patients, especially those with AIDS [33,34]. NET formation was not induced when neutrophils were exposed to the wild-type strain (B3501 strain) of Cryptococcus neoformans in vitro but was induced when exposed to an acapsular mutant (CAP67 mutant strain) of Cryptococcus neoformans [35]. NET formation by an acapsular mutant (CAP67 mutant strain) is ROS and PAD-4 dependent [35].

\section{NETs and Parasites}

Some researchers have investigated the role of NET formation in parasitic infections. Baker et al. found that children infected with Plasmodium falciparum induce NET formation [36]. NET formation 
Page 3 of 5

was also observed with Leishmania parasites. Neutrophils incubated with Leishmania were shown to form NETs to trap Leishmania [37]. The parasitic molecule Promastigote LPG induced NETs in a dosedependent manner, and the NET component histone killed the pathogen [37]. Toxoplasmosis is a worldwide infection that affects at least a third of the world's population by the intracellular parasite Toxoplasma gondii [38]. Toxoplasma gondii elicits NET formation by both human and mice neutrophils [39]. Activating the Raf-MEK-ERK signalling pathway is required in NET formation [40]. Blocking the Raf-MEK-ERK signalling pathway by U0126, a well-known chemical inhibitor of MEK1/2, prevents Toxoplasma-induced NET formation [39].

\section{NETs and Autoimmune Disorders}

Countless studies have determined the impact of NETs during infections. However, many studies show the role of NETs in inflammatory and autoimmune diseases, including rheumatoid arthritis, lupus, vasculitis, gout, antiphospholipid antibody syndrome (APS) and diabetes.

\section{Systemic Lupus Erythematosus (SLE, lupus)}

Systemic lupus erythematosus is an autoimmune disease with various clinical symptoms. Autoimmune diseases occur when the immune system produces antibodies against the body's cells. SLE can affect most tissue such as the skin, joints, brain, lung, kidney, and heart [41]. Neutrophils isolated from healthy individuals exposed to SLE plasma have shown to form NETs [42]. NET formation by neutrophils during infections is a crucial process for protecting the body from invading pathogens. However, the extravagant NET formation may cause tissue inflammation and damage to the organs [43]. DNasel is shown to potent for NET degradation [44]. Hakkim et al. found that sera from SLE patients contains DNasel inhibitors and can degrade NETs [44]. However, they demonstrated that some sera isolated from some SLE patients contained high titration of antibodies that bind to NETs and protected them from DNase [44]. Several other reports have demonstrated the reduced ability of SLE patients to degrade NETs $[45,46]$.

\section{Rheumatoid Arthritis}

Rheumatoid arthritis is a serious autoimmune disease that causes inflammation of small and large joints, which leads to disability and discomfort [47]. Synovial fluid and peripheral blood caused by rheumatoid arthritis display significant NET formation without stimuli when compared to peripheral blood neutrophils or synovial fluid neutrophils isolated from osteoarthritis patients. Besides, on stimulation with lipopolysaccharide (LPS), rheumatoid arthritis synovial fluid and peripheral blood were shown to enhance NET formation compared to neutrophils isolated from healthy individuals and osteoarthritis patients [48]. Formation of autoantibodies to citrullinated antigens (ACPA) is considered as a key pathogenic phenomenon in RA. Serum and synovial fluid isolated from RA with high levels of ACPA have shown to induce a significant amount of NETs in control and RA neutrophils when compared to control sera or osteoarthritis synovial fluid [48]. Chowdhury et al demonstrated the substantial increase in NET formation in rheumatoid arthritis [49]. To sum up, a considerable amount of NETs has been found in RA patients and this may lead to inflammation of the joints and may cause tissue damage.

\section{Gout}

Gout is a form of arthritis caused by an increase in the amount of monosodium urate (MSU) crystals in the joints, which leads to inflammation [50]. Mitroulis et al. found that monosodium urate (MSU) crystals induce NET formation. Also, control neutrophils treated with synovial fluid from inflamed joints form extensive NETs, suggesting that the inflammatory environment of gout induces NET release [51]. NET formation in gout has been shown to trap MSU crystals and prevent further complications caused by the MSU crystals [52]. Further studies need to be conducted to confirm the involvement of NET release in the pathogenesis of inflammatory arthritis.

\section{Vasculitis}

The pathogenesis of NET formation varies between different forms of vasculitis. However, NETs play a role in small-vessel vasculitis (SVV) [53]. Neutrophils stimulated with antineutrophil cytoplasm autoantibodies (ANCAs), which are linked to SVV, have been found to form NETs composed of proteinase-3 (PR3) and myeloperoxidase (MPO) [54]. One report that shows the relationship between NETs with vasculitis suggested that accumulated NETs and circulating NETs components in inflamed kidneys trigger vasculitis in individuals with SVV [54].

\section{Antiphospholipid Antibody Syndrome (APS)}

Antiphospholipid antibody syndrome (APS) is an autoimmune disease associated with elevated titres of antiphospholipid antibodies (aPL) [55]. Cell-free DNA and NETs have been observed in the plasma of APS patients. Purified IgG and the sera of APS patients have been found to stimulate NETs from control neutrophils. NET has also been released by monoclonal human antiphospholipid antibodies (aPL), especially those targeting $\beta 2$ GPI. Inhibiting TLR-4 signalling and ROS formation could abrogate APS NETs [55]. Besides, a recent study suggested that antiphospholipid antibodies prime circulating neutrophils to accelerate thrombosis. Meng et al. observed extravagant thrombosis in mice treated with APS IgG than in mice treated with control IgG. The thrombus in mice treated with APS IgG was decorated with the NET marker citrullinated histone H3 (Cit-H3) [56].

\section{Diabetes}

The role of neutrophil extracellular traps (NETs) in diabetes was determined in a handful of reports. Stimulated neutrophils from patients with type 1 and type 2 diabetic human and mice induce NET formation [57]. Interestingly, neutrophils from diabetic human are more susceptibility to NETosis than non-diabetic human, this might be due to the elevated blood glucose level in diabetic human than nondiabetic [57-59]. Peptidylarginine deiminase 4 (PAD4) has been known to be essential for chromatin decondensation during the process of NETosis [60]. Inhibition of PAD4 activity with Cl-amidine and PAD4-/- mice facilitates wound healing and proving that NETs could interfere with wound healing $[57,59]$.

\section{Conclusion}

Extensive studies of NET release in vitro and in vivo against infection and during inflammation have improved our understanding of most of their roles. These studies have suggested that NET formation can occur via distinct mechanisms mostly led by the type of stimuli. Further, the excessive NET formation is involved in the 
aggravation of the disease in some conditions. The inhibition of NET release could help to determine further complications of excessive NET formation in some disorders, and this could be a therapeutic strategy in the future.

\section{Acknowledgement}

I thank King Khalid University for providing me with the facilities to write this paper. I am grateful to my colleague Jaya Shankar Tedla for his honest advice.

\section{References}

1. Brinkmann V, Reichard U, Goosmann C, Fauler B, Uhlemann Y, et al. (2004) Neutrophil Extracellular Traps Kill Bacteria. Science 303: 1532-1535.

2. Yipp BG, Kubes P (2013) NETosis: How vital is it? Blood 122: 2784-2794.

3. Mohanty T, Sjögren J, Kahn F, Abu-Humaidan AH, Fisker N, et al. (2015) A novel mechanism for NETosis provides antimicrobial defense at the oral mucosa. Blood 126: 2128-2137.

4. Brinkmann V, Zychlinsky A (2012) Neutrophil extracellular traps: Is immunity the second function of chromatin? J Cell Biol 198: 773-783.

5. Steinberg BE, Grinstein S (2007) Unconventional roles of the NADPH oxidase: signaling, ion homeostasis, and cell death. Sci STKE 379: pe11pe11.

6. Fuchs TA, Abed U, Goosmann C, Hurwitz R, Schulze I, et al. (2007) 'Novel cell death program leads to neutrophil extracellular traps. J Cell Biol 176: 231-241.

7. Papayannopoulos V, Metzler KD, Hakkim A, Zychlinsky A (2010) Neutrophil elastase and myeloperoxidase regulate the formation of neutrophil extracellular traps. J Cell Biol 191: 677-691.

8. Pilsczek FH, Salina D, Poon KK, Fahey C, Yipp BG, et al. (2010) A novel mechanism of rapid nuclear neutrophil extracellular trap formation in response to Staphylococcus aureus. J Immunol 185: 7413-7425.

9. Clark SR, Ma AC, Tavener SA, McDonald B, Goodarzi Z, et al. (2007) Platelet TLR4 activates neutrophil extracellular traps to ensnare bacteria in septic blood. Nat Med 13: 463-469.

10. Yipp BG, Petri B, Salina D, Jenne CN, Scott BN, et al. (2012) Infectioninduced NETosis is a dynamic process involving neutrophil multitasking in vivo. Nat Med 18: 1386-1393.

11. Parker H, Albrett AM, Kettle AJ, Winterbourn CC (2012) Myeloperoxidase associated with neutrophil extracellular traps is active and mediates bacterial killing in the presence of hydrogen peroxide. J Leukoc Biol 91: 369-376.

12. Beiter K, Wartha F, Albiger B, Normark S, Zychlinsky A, et al. (2006) An endonuclease allows Streptococcus pneumoniae to escape from neutrophil extracellular traps. Current Biology 16: 401-407.

13. Puyet A, Greenberg B, Lacks SA (1990) Genetic and structural characterization of endA. A membrane-bound nuclease required for transformation of Streptococcus pneumoniae. J Mol Biol 213: 727-738.

14. Lyautey E, Lapen DR, Wilkes G, McCleary K, Pagotto F, et al. (2007) Distribution and characteristics of Listeria monocytogenes isolates from surface waters of the South Nation River watershed, Ontario, Canada. Appl Environ Microbiol 73: 5401-5410.

15. Munafo DB, Johnson JL, Brzezinska AA, Ellis BA, Wood MR, et al. (2009) DNase $\mathrm{i}$ inhibits a late phase of reactive oxygen species production in neutrophils. J Innate Immun 1: 527-542.

16. Braian C, Hogea V, Stendahl O (2013) Mycobacterium tuberculosisinduced neutrophil extracellular traps activate human macrophages. J Innate Immun 5: 591-602.

17. McCormick A, Heesemann L, Wagener J, Marcos V, Hartl D, et al. (2010) NETs formed by human neutrophils inhibit growth of the pathogenic mold Aspergillus fumigatus. Microbes Infect 12: 928-936.
18. Urban CF, Reichard U, Brinkmann V, Zychlinsky A, et al. (2006) Neutrophil extracellular traps capture and kill Candida albicans yeast and hyphal forms. Cell Microbiol 8: 668-676.

19. Johnson CJ, Kernien JF, Hoyer AR, Nett JE (2017) Mechanisms involved in the triggering of neutrophil extracellular traps (NETs) by Candida glabrata during planktonic and biofilm growth. Sci Rep 7: 13065.

20. King P (2012) Haemophilus influenzae and the lung (Haemophilus and the lung). Clinical and Translational Medicine 1: 10.

21. Van Eldere J, Slack MP, Ladhani S, Cripps AW (2014) Non-typeable Haemophilus influenzae, an under-recognised pathogen. Lancet Infect Dis 14: 1281-1292.

22. Juneau RA, Pang B, Weimer KE, Armbruster CE, Swords WE (2011) Nontypeable haemophilus influenzae initiates formation of neutrophil extracellular traps. Infect Immun 79: 431-438.

23. Möllerherm H, Neumann A, Schilcher K, Blodkamp S, Zeitouni NE, et al. (2015) Yersinia enterocolitica-mediated degradation of neutrophil extracellular traps (NETs). FEMS Microbiology Lett 362: fnv192.

24. How KY, Song KP, Chan KG (2016) Porphyromonas gingivalis: An Overview of Periodontopathic Pathogen below the Gum Line. Front Microbiol 7: 53.

25. Delbosc S, Alsac JM, Journe C, Louedec L, Castier Y, et al. (2011) Porphyromonas gingivalis Participates in Pathogenesis of Human Abdominal Aortic Aneurysm by Neutrophil Activation. Proof of Concept in Rats. PLoS ONE 6: e18679.

26. Seper A, Hosseinzadeh A, Gorkiewicz G, Lichtenegger S, Roier S, et al. (2013) Vibrio cholerae Evades Neutrophil Extracellular Traps by the Activity of Two Extracellular Nucleases. PLoS Pathogens 9: e1003614.

27. Tripathi S, Verma A, Kim EJ, White MR, Hartshorn KL (2014) LL-37 modulates human neutrophil responses to influenza A virus. J Leukoc Biol 96: 931-938.

28. Moreno-Altamirano MMB, Rodríguez-Espinosa O, Rojas-Espinosa $\mathrm{O}$ Pliego-Rivero B, Sánchez-García FJ (2015) Dengue Virus Serotype-2 Interferes with the Formation of Neutrophil Extracellular Traps. Intervirology 58: 250-259.

29. Saitoh T, Komano J, Saitoh Y, Misawa T, Takahama M, et al. (2012) Neutrophil extracellular traps mediate a host defense response to human immunodeficiency virus-1. Cell Host Microbe 12: 109-116.

30. Cortjens B, de Boer OJ, de Jong R, Antonis AF, Sabogal Piñeros YS, et al. (2016) Neutrophil extracellular traps cause airway obstruction during respiratory syncytial virus disease. J Pathol 238: 401-411.

31. Kim J, Sudbery P (2011) Candida albicans, a major human fungal pathogen. J Microbiol 49: 171-177.

32. Bruns S, Kniemeyer O, Hasenberg M, Aimanianda V, Nietzsche S, et al. (2010) Production of extracellular traps against Aspergillus fumigatus in vitro and in infected lung tissue is dependent on invading neutrophils and influenced by hydrophobin RodA. PLoS Pathogens 6: e1000873.

33. Röhm M, Grimm MJ, D'Auria AC, Almyroudis NG, Segal BH, et al. (2014) NADPH oxidase promotes neutrophil extracellular trap formation in pulmonary aspergillosis. Infect Immun 82: 1766-1777.

34. Warkentien T, Crum-Cianflone NF (2010) An update on cryptococcus among HIV-infected patients. Int J STD AIDS 21: 679-684.

35. Rocha JDB, Nascimento MT, Decote-Ricardo D, Côrte-Real S, Morrot A, et al. (2015) Capsular polysaccharides from Cryptococcus neoformans modulate production of neutrophil extracellular traps (NETs) by human neutrophils. Sci Rep 5: 8008.

36. Baker VS, Imade GE, Molta NB, Tawde P, Pam SD, et al. (2008) Cytokineassociated neutrophil extracellular traps and antinuclear antibodies in Plasmodium falciparum infected children under six years of age. Malar J $7: 41$.

37. Guimaraes-Costa AB, Nascimento MT, Froment GS, Soares RP, Morgado FN, et al. (2009) Leishmania amazonensis promastigotes induce and are killed by neutrophil extracellular traps. Proceedings of the National Academy of Sciences 106: 6748-6753.

38. Saadatnia G, Golkar M (2012) A review on human toxoplasmosis. Scandinavian Journal of Infectious Diseases 44: 805-814. 
Page 5 of 5

39. Abi Abdallah DS, Lin C, Ball CJ, King MR, Duhamel GE, et al. (2012) 'Toxoplasma gondii Triggers Release of Human and Mouse Neutrophil Extracellular Traps. Infect Immun 80: 768-777.

40. Hakkim A, Fuchs TS, Martinez NE, Hess S, Prinz H, et al. (2011) Activation of the Raf-MEK-ERK pathway is required for neutrophil extracellular trap formation. Nat Chem Biol 7: 75-77.

41. Cunha JS, Gilek-Seibert K (2016) Systemic Lupus Erythematosus: A review of the clinical approach to diagnosis and update on current targeted therapies. R I Med J 99: 23-27.

42. Van Avondt K, Fritsch-Stork R, Derksen RH, Meyaard L (2013) Ligation of signal inhibitory receptor on leukocytes-1 suppresses the release of neutrophil extracellular traps in systemic lupus erythematosus. PLoS ONE 8: e78459.

43. Branzk N, Papayannopoulos V (2013) Molecular mechanisms regulating NETosis in infection and disease. Semin Immunopathol 35: 513-530.

44. Hakkim A, Fürnrohr BG, Amann K, Laube B, Abed UA, et al. (2010) Impairment of neutrophil extracellular trap degradation is associated with lupus nephritis. Proc Natl Acad Sci U S A 107: 9813-9818.

45. Leffler J, Martin M, Gullstrand B, Tyden H, Lood C, et al. (2012) Neutrophil extracellular traps that are not degraded in systemic lupus erythematosus activate complement exacerbating the disease. J Immunol 188: 3522-3531.

46. Leffler J, Gullstrand B, Jönsen A, Nilsson JA, Martin M, et al. (2013) Degradation of neutrophil extracellular traps co-varies with disease activity in patients with systemic lupus erythematosus. Arthritis Res Ther 15: R84.

47. Arora S, Rafiq A, Jolly M (2016) Management of rheumatoid arthritis: Review of current guidelines. Journal of Arthroscopy and Joint Surgery 3: 45-50.

48. Khandpur R, Carmona-Rivera C, Vivekanandan-Giri A, Gizinski A, Yalavarthi S, et al. (2013) NETs are a source of citrullinated autoantigens and stimulate inflammatory responses in rheumatoid arthritis. Sci Transl Med 5: $178 \mathrm{ra} 40$

49. Sur Chowdhury C, Giaglis S, Walker UA, Buser A, Hahn S, et al. (2014) 'Enhanced neutrophil extracellular trap generation in rheumatoid arthritis: Analysis of underlying signal transduction pathways and potential diagnostic utility. Arthritis Res Ther 16: R122.

50. Ragab G, Elshahaly M, Bardin T (2017) Gout: An old disease in new perspective - A review. J Adv Res 8: 495-511.

51. Mitroulis I, Kambas K, Chrysanthopoulou A, Skendros P, Apostolidou E, et al. (2011) Neutrophil extracellular trap formation is associated with IL-1 $\beta$ and autophagy-related signaling in gout. PLoS ONE 6: e29318.

52. Schett G, Schauer C, Hoffmann M, Herrmann M (2015) Why does the gout attack stop? A roadmap for the immune pathogenesis of gout. RMD Open 1: e000046.

53. Soderberg D, Segelmark M (2018) Neutrophil extracellular traps in vasculitis, friend or foe? Curr Opin Rheumatol 30: 16-23.

54. Kessenbrock K, Krumbholz M, Schönermarck U, Back W, Gross WL, et al. (2009) Netting neutrophils in autoimmune small-vessel vasculitis. Nat Med 15: 623-625.

55. Yalavarthi S, Gould TJ, Rao AN, Mazza LF, Morris AE, et al. (2015) Release of neutrophil extracellular traps by neutrophils stimulated with antiphospholipid antibodies: a newly identified mechanism of thrombosis in the antiphospholipid syndrome. Arthritis Rheumatol 67: 2990-3003.

56. Meng H, Yalavarthi S, Kanthi Y, Mazza LF, Elfline MA, et al. (2017) In Vivo role of neutrophil extracellular traps in antiphospholipid antibodymediated venous thrombosis. Arthritis Rheumatol 69: 655-667.

57. Wong SL, Demers M, Martinod K, Gallant M, Wang Y, et al. (2015) Diabetes primes neutrophils to undergo NETosis, which impairs wound healing. Nat Med 21: 815-819.

58. Carestia A, Frechtel G, Cerrone G, Linari MA, Gonzalez CD, et al. (2016) NETosis before and after hyperglycemic control in type 2 diabetes mellitus patients. PLoS ONE 11: e0168647.

59. Fadini GP, Menegazzo L, Rigato M, Scattolini V, Poncina N, et al. (2016) NETosis delays diabetic wound healing in mice and humans. Diabetes 65: 1061-1071.

60. Wang Y, Li M, Stadler S, Correll S, Li P, et al. (2009) Histone hypercitrullination mediates chromatin decondensation and neutrophil extracellular trap formation. J Cell Biol 184: 205-213. 